\title{
La diáspora india, su relación con el país de origen y residencia: Panorama de los estudios realizados
}

\author{
Lía Rodríguez de la Vega' \\ Universidad Nacional de Lomas de Zamora, Universidad de Palermo (Argentina)
}

Recibido: diciembre 4 de 2015. Revisado: enero 16 de 2016. Aceptado: febrero 10 de 2016

Referencia formato APA: Rodríguez-de la Vega, L. (2016). La diáspora india, su relación con el país de origen y residencia: Panorama de los estudios realizados. Rev. Guillermo de Ockham, 14(1), 89-97. doi: http://dx.doi.org/10.21500/22563202.2309

\section{Resumen}

De acuerdo con lo señalado por la Organización Internacional para las Migraciones, se estima en 214 millones las personas que viven fuera de su país de origen. En ese marco, la denominada diáspora india cuenta con más de 20 millones de personas fuera de India. Basado en las diásporas, Sheffer apunta que las redes creadas por diásporas étnicas forman parte de una relación triádica que comprende a la diáspora misma, su país de origen y el país de residencia. Señala, además, que esa relación deviene un aspecto integral y permanente del flujo de política nacional e internacional. Al hilo de lo anterior, el presente trabajo aborda la bibliografía académica sobre la relación triádica mencionada y sus componentes, en el marco del estudio de las migraciones en el ámbito internacional, desde 2000. Se revisan los aportes, los elementos que se van a profundizar y los vacíos y se concluye con la propuesta de un abordaje interdisciplinario del fenómeno.

Palabras clave: Migraciones, relaciones internacionales, diáspora india, país de residencia, país de origen

\section{The Indian diaspora, its relationship with the country of origin and of residence: state of the art}

Abstract

As enunciated by the International Organization for Migration/IOM, it is estimated that at present there are 214 million people living outside their country of origin. In this context, the so-called Indian diaspora has more than 20 million people, outside India. Strictly regarding diasporas, Sheffer (1986) remarks that the networks created by ethnic diasporas form part of a three-way relationship that includes the diaspora itself, its country of origin and of residence, pointing also that this relationship becomes an integral and permanent aspect of the flow of national and international politics. Considering the above mentioned, the present work approaches the academic literature produced on the aforementioned three-way relationship and its components, within the framework of the study of migration in the international field, since 2000. Contributions and topics worth further research are reviewed. It concludes with the proposal of an interdisciplinary approach of the phenomenon.

Keywords: Migration, international relations, Indian diaspora, country of residence, country of origin

1. Experta en hinduismo yoga. Licenciada en Estudios Orientales (Usal). Doctora en Relaciones Internacionales (Usal). Estudios postdoctorales en la Universidad Federal de Río Grande do Sul, UFRGS (Porto Alegre, Brasil). Responsable de las áreas de Asia y África de Unicom, Facultad de Ciencias Sociales (UNLZ), investigadora del Centro de Investigaciones en Ciencias Sociales de la Facultad de Ciencias Sociales, Universidad de Palermo y Coordinadora del Grupo de Trabajo sobre India, Comité de Asuntos Asiáticos, C.A.R.I. Virrey Loreto 2650- 9. "B”, Ciudad Autónoma de Buenos Aires. Código Postal: 1426.E-mail: liadelavega@yahoo.com 


\section{A diáspora india, sua relação com o pais de origem e residência: Panorama dos estudos realizados}

\section{Resumo}

De acordo com as conclusôes da Organização Internacional para as Migraçóes / IOM, estima-se que existam 214 milhóes de pessoas que vivem fora do seu país de origem. Neste contexto, a chamada diáspora indiana tem mais de 20 milhôes de pessoas fora da Índia. Em resposta precisamente diásporas, Sheffer (1986) aponta que as redes criadas pelas diásporas étnicas são parte de uma relação triádica que compreende da própria diáspora, seu país de origem e de residência, também apontar que essa relaçáo se torna um aspecto integral e permanente o fluxo de políticas domésticas e internacionais. Diante do exposto, este artigo aborda a literatura académica produzida sobre a relação triádica mencionado e seus componentes, no estudo da migração a nível internacional, desde 2000, as contribuiçôes são revistos para aprofundar os elementos e as lacunas e conclui-se com a proposta de uma abordagem interdisciplinar para o fenômeno.

Palvras-chave: Migração, relações internacionais, diáspora indiana, país de residência, país de origem

\section{Introducción}

De acuerdo con la Organización Internacional para las Migraciones (OIM) (2010), se estima que 214 millones de personas residen en el presente fuera de su país de origen. El fenómeno migratorio supera el plano nacional, influye en las relaciones interestatales y de distintos actores no estatales y se constituye, incluso, en instrumento de la política exterior con efectos interiores y exteriores en los países emisores y en los receptores. Las comunidades emigradas bajo la denominación de diáspora, aparecen de hecho entre los actores no estatales, que ejercen una influencia significativa a escala nacional y global (Gupta, 2013).

Las redes creadas por diásporas étnicas tienen características particulares dado que forman parte de una relación triádica que comprende el país de origen, la diáspora misma y los países de residencia (Sheffer, 1986). Esta relación pone en evidencia dinámicas articuladoras de lo global y lo local, que abarcan distintas temáticas y deviene un aspecto integral y hasta permanente del flujo de política de ambos niveles. Las diásporas étnicas, asentadas en nuevos países como grupos minoritarios, preservan su identidad étnica y su solidaridad comunal, y este último elemento permite mantener y promocionar el contacto con sus elementos activistas, que a su vez poseen diversos significados para los distintos componentes de la relación triádica.

Así, la consideración de los grupos diaspóricos ha derivado en un alcance interdisciplinario en el campo de las ciencias sociales, con conexiones teóricas entre distintas disciplinas.

El arribo de un nuevo orden trajo consigo el reconocimiento de los académicos de una gran variedad de nuevos actores -sobre todo no estatales-anteriormente ignorados en política internacional (Mitchell, 2012). Tal el caso de las migraciones, que captaron nuevamente la atención tras los incidentes de septiembre de 2001, lo cual derivó en trabajos enfocados en la perspectiva securitaria. Finalmente, apunta que la temática migratoria es considerada como de "alta política" en la nueva agenda política y si bien se han registrado avances en su estudio en el marco específico de las relaciones internacionales, la comprensión de sus implicancias políticas es aún limitada.

Con base en lo mencionado, el presente trabajo aborda la bibliografía académica producida sobre la relación triádica aludida y sus componentes, en el marco de la consideración de las migraciones en el ámbito internacional, desde 2000.

\section{Estudios sobre la relación triádica país de origen-diáspora-país de residencia y sus componentes}

\section{Abordajes sobre el pais de origen}

Leclerc (2004) considera la invención de la diáspora india como tal y señala que el uso de la idea de una "diáspora" para aludir a los emigrados de la India aparece en un artículo de Bharati (1976) sin que el término se mencionara de manera explícita. Ya en 2004, Jayaram haría pleno uso del vocablo. Ese reconocimiento de la categoría "diáspora india" se reforzaría en conferencias internacionales que abordaron la temática, antecedidas por la creación de un centro para su estudio en la Universidad de Hyderabad, India, en 1996 y la creación por el gobierno indio de un comité de alto nivel para su análisis. 
Rodríguez de la Vega (2014) hace una aproximación a la diáspora india en el marco de la articulación entre lo local y lo global y toma en cuenta los tres elementos de la triada, para finalmente concluir que la enunciación de la "diáspora india" desde la perspectiva considerada, da cuenta de esa articulación-que por lo demás es una tarea permanente- en el contexto de la resignificación identitaria de la "indianidad", sus alcances en la configuración del espacio y el tiempo (entre India y la diáspora) y la capacidad de influencia que ello conlleva.

Oonk (2007) por su parte, en su compilación de textos referidos a diferentes segmentos de la diáspora en la que rescata sus heterogeneidades, menciona tres perspectivas sobre el concepto de diáspora: 1 . la que alude al marco analítico de los académicos; 2. la sostenida por el Gobierno de la India ${ }^{2}$ que establece una categorización específica de las personas (la diáspora incluye a los ciudadanos indios que no residen en India, a las personas de origen indio que han tomado otra nacionalidad y a las personas que técnicamente pueden calificarse como apátridas de origen indio, que no cuentan con documentación que permita acreditar su origen indio, de acuerdo con lo señalado por Sharma, s.f.); y 3. la perspectiva de los migrantes mismos.

Hercog y Sieggel (2010) abordan las políticas desarrolladas por el Gobierno indio en torno a la diáspora (creación de un comité de alto nivel sobre la diáspora india para su estudio; establecimiento del Pravasi Bharatiya Divas - día de la diáspora india-; aceptación de una ciudadanía de ultramar para algunos países de residencia, y creación del Ministerio para los Asuntos de los Indios en el Extranjero). Naujoks (2010) afronta el cambio tanto en las políticas estatales indias como (según lo que sostiene) en los abordajes de investigación indios sobre la diáspora india, estableciendo de esta manera un paralelo poderconocimiento que cabe seguir profundizando. Singh (2012) se concentra en la perspectiva política india para el trabajo con la diáspora, en lo que concierne al desarrollo de India, y Lum (2012) se focaliza en las políticas desarrolladas por India y China, países relevantes en el ámbito internacional, hacia sus respectivas diásporas (ambas entre las mayores del mundo).

Murti (2013) analiza el uso de los medios digitales para captar a la diáspora en el marco de la diplomacia pública india, mediante el estudio del sitio web del Centro de Facilitación de los Indios de Ultramar (oIfC), del cual toma un modelo que considera tres niveles de diploma- cia pública: monólogo, diálogo y colaboración. Khana (2005), por su parte, alude a la diáspora como un potencial multiplicador de la fuerza diplomática de India y traduce el cosmopolitismo indio como bollystan, reino de raíces indias plantadas en todos lados que emerge orgánicamente como la mezcla de etnocomercio y un nuevo modelo de soberanía que trasciende lo geográfico.

Mason (2013) apunta la importancia de los indios residentes en Arabia Saudita, sus remesas, su situación y el accionar político del gobierno indio sobre ellos, en consideración de una alianza estratégica entre ambos países y Abraham (2012) estudia la dinámica entre India y la diáspora asentada en la zona del Golfo Pérsico, con base en el poder blando y la diplomacia pública en juego.

En cuanto a las repercusiones de la diáspora en la economía del país de origen, Roy y Banerjee (2007) señalan que la inversión extranjera directa desempeñó un papel de mayor importancia en China que en India y que la reorientación de los hechos en este sentido tendrá que ver de modo fundamental con la función que habrá de cumplir el oIfc. Zhiqun Zhu (2007) compara el efecto de las diásporas china e india asentadas en Estados Unidos sobre el ámbito económico de sus países de origen y señala que la globalización profundizará la migración internacional, que la "fuga de cerebros" ha sido reemplazada por la "circulación de cerebros" y que ello ha devenido un fenómeno único en la política económica internacional actual que merece mayor estudio.

Leclerc (2008) aborda la manera como India devino central en la industria tecnológica global mediante la provisión de recursos humanos, accediendo así a un nuevo lugar en la división internacional del trabajo con un papel destacado de la diáspora en la fase de outsourcing internacional desde los ańos ochenta. Por su parte, Van Hear, Pieke, Vertovec, Lindley, Jettinger y Balarajan (2004) atienden al aporte que distintas diásporas asentadas en el Reino Unido hacen a sus países de origen en cuanto al desarrollo y la reducción de la pobreza. Señalan los envíos de dinero de los emigrados indios para mejoras en infraestructura social y religiosa, ayuda en desastres, etc. Otros estudios relativos a la dinámica económica entre la diáspora e India son los de Kapoor (2003); Pandey, Aggarwal, Devane y Kuznetso (2004); Singh (2005); Chanda y Sreenivasan (2006); Balasubramanyam (2007); Cnada (2008) y Chand (2009).

2. El gobierno indio entiende a la diáspora como " [... un término genérico que describe personas emigradas de territorios que están actualmente dentro de los límites de la República de la India. Se refiere también a sus descendientes". Señala, asimismo, que estas personas "residentes en tierras lejanas [...] retuvieron su ligazón emocional, cultural y espiritual con su país de origen” (The Indian Diaspora, párr. 1 y 2). 
Therwath (2010) especifica otros impactos de los emigrados en el país de origen, en donde son mostrados como modelos emprendedores cuya ejemplaridad resulta atestiguada por su éxito financiero. Agrega que los NRI se convirtieron en la síntesis de la "indianidad", vistos como instrumentos de la modernidad en India, al mismo tiempo que factores del reconocimiento internacional de este país, en occidente Kapur (2010), mientras tanto, examina el caso del impacto del segmento de la diáspora india asentado en los Estados Unidos sobre la situación doméstica, en cuanto al fomento de la violencia política y al desarrollo de la filantropía.

Niumai (2009) también aborda la filantropía en el mismo segmento de la diáspora, al señalar la necesidad de dotar a esa filantropía de una influencia estratégica sobre los programas de desarrollo en India con sus ideas innovadoras, mientras Heath y Mathur (2011) reúnen trabajos de distintos autores que abordan la dinámica entre la diáspora y el país de origen, en cuestiones relativas al comunalismo. Otros trabajos que vale mencionar sobre esta temática son los de Bhatt y Mukta (2000); Pradash (2003); Bal y Sinha-Kerkhof (2007); Gayer (2007); Therwath (2007), y Kinnvall y Svensson (2010).

Por su parte, Mohan y Chauhan (2015) tratan la cuestión de la diáspora india en la política exterior del Primer Ministro Modi (elegido en 2014) y señalan que él tiene la oportunidad de influir en la diáspora de Asia del sur para que impacte en sus países de origen en términos de moderación política, reconciliación regional y modernización del subcontinente. Agregan que resulta de interés el rol potencial de la diáspora en la transformación de la relación con países anglo-americanos, y advierten sobre el peligro de la réplica diaspórica de los disturbios domésticos.

\section{Abordajes sobre la dinámica de la diáspora}

En paralelo con los estudios sobre el país de origen, la diáspora, al tiempo que alcanzaba distintos destinos, fue también organizándose en distintas redes creadas voluntariamente y convirtiéndose en terreno particular de distintas acciones rescatadas a su vez por otros estudios.

Bhat y Narayan (2010) consideran el fenómeno de las diásporas en el marco del transnacionalismo, y subrayan en su relación con el país de origen y su conexión con otras comunidades dispersas en el mundo, sumado a los sentimientos comunitarios a través de diferentes países, las diásporas constituyen lo descrito bajo el término transnacionalismo. En ese marco, mencionan distintas organizaciones que dan cuenta del diverso espectro identitario indio, entre las que se cuentan la Organización Global para Personas de Origen Indio (Global Organization of People of Indian Origin -Gopio-), propuesta panindia a nivel global; la Federación Mundial Telegu (WTF) y la Organización Mundial Sikh. En una dirección similar, Mulloo (2007) avanza en más detalles de Gopio y su estructura organizativa.

Agarwala (2012) sostiene que las comunidades diaspóricas y el Gobierno de la India modificaron su relación desde mediados de la década de 1980. Así, mientras India iniciaba el desarrollo de políticas específicas para profundizar esta relación, en los Estados Unidos, por ejemplo, se experimentó un aumento súbito del número de organizaciones en el ámbito de la diáspora (de las que provee un inventario). Agrega que el interés del Gobierno indio se basó en la visión del potencial de la diáspora para el desarrollo indio, en el hecho de que la globalización de la economía india redujera los prejuicios anteriores en contra de los emigrados y en el ejemplo de las comunidades diaspóricas chinas en su colaboración con la expansión de la economía de su país de origen.

Por su parte Gautam (2013) da cuenta de organizaciones de la diáspora india en Europa desde tiempo atrás, tales como la Sociedad Sikh,Khalsa Jatha (1908), la Asociación Hindú de Europa (1935), otras sociedades islámicas y parsis, la Sociedad India de Bienestar (Iws) y la Fundación para las Elecciones Críticas de India (FCCI). Mientras analiza cuestiones relativas a las asociaciones, aborda de manera particular la dinámica de esta diáspora en Alemania y Holanda y considera posibles vías para contribuir a la relación entre Europa e India.

Lacroix (2011) analiza las características de las asociaciones de migrantes en el Reino Unido mediante dos casos de la comunidad india asentada allí (el Grupo Sikh de Derechos Humanos y la Asociación de Trabajadores Indios de Birmingham) y dos de la comunidad polaca. Su trabajo reporta el compromiso ocasional de las asociaciones con algunas actividades en el exterior, señala la interconexión de organizaciones a través de las fronteras y su contribución a entretejer lo que puede ser denominado campos de organizaciones transnacionales a nivel global.

Reddy (2015) aborda la dinámica de los movimientos sociales en la diáspora india -otro fenómeno transnacional de interés que puede tener ramificaciones políticas- $y$ resalta que la transnacionalidad intrínseca a la identidad de las diásporas las aleja del país de origen y de los de residencia y las sitúa en una localidad transnacional donde los movimientos sociales en la diáspora ganan notoriedad. Tal posicionamiento constituye para los países de residencia gran desafío frente a las diásporas, limitado, claro está, 
por su identidad híbrida y sus lealtades divididas: al país de origen $o$ al de residencia.

Omer (2011) considera la religión en su confluencia con lo político y el nacionalismo en la diáspora india, al afrontar esta última junto a los emigrados judíos asentados en Estados Unidos. Señala que el activismo diaspórico del hindutva (nacionalismo hindú) manifestado a través del Comité de Asuntos Públicos Americano-israelí (Aipac), abarca diversos asuntos y hace eco en algunos casos con el discurso general americano sobre el terrorismo yihadista. Ello guarda relación con acciones de otros agentes que evidencian la imaginación y codificación del hinduismo como una comunidad transnacional y homogénea. Concluye proponiendo una fertilización cruzada entre estudios de las diásporas en las relaciones internacionales, estudios culturales y sobre la paz.

Mohammad-Arif (2000; 2007) atiende la reconstrucción de las identidades hindú e islámica entre las diásporas del sur de Asia en Estados Unidos y especifica la polarización entre ambas por diversos motivos que aluden al país de residencia y a los de origen (India y Paquistán) y a la compleja renegociación identitaria de ambos grupos. Resalta, asimismo, el funcionamiento de grupos religiosos como grupos étnicos en atención a que la etnicidad es considerada como el único marcador identitario válido a nivel oficial en el país de residencia.

Finalmente, cabe mencionar a Varadarajan (2010), quien postula que la novedad del fenómeno de la diáspora no niega los lazos transnacionales existentes anteriormente. Para este autor, dicha novedad reside en el hecho de que la producción de esa "domesticidad fuera de India" descansa en la constitución de las diásporas como sujetos de una nación expandida y difusa territorialmente, lo cual ocurre en un momento en que el Estado asume un carácter y una naturaleza nuevos.

\section{Abordajes sobre los países de residencia}

Se encuentran también trabajos sobre la repercusión de segmentos específicos de la diáspora india en los países en los que se asientan.

En esta dirección, Janardhanan (2013) aborda la participación política de la diáspora india asentada en los Estados Unidos y analiza la manera como los obstáculos y prejuicios iniciales en el país de residencia sirvieron de catalizadores de la actividad política de la comunidad, sin olvidar los desafíos que enfrenta. Resulta de interés su consideración de que el activismo político diaspórico transnacional no es un fenómeno nuevo, y en ese sentido recuerda los ecos del incidente del barco Komagata Maru - a cuyos pasajeros indios les fuera negada la entrada en Canadá, en 1914- y el surgimiento del conocido movimiento Gadar, que retomaba en la diáspora las ambiciones libertarias indias del Gobierno británico.

Sobre el mismo segmento de la diáspora, Gottschlisch (2008) explora elementos relacionados a su fuerza política. Sostiene que esta aumenta en el país de residencia por medio del uso de cuatro recursos principales: influencia a través del voto y la opinión pública; influencia a través de la información e instituciones; influencia a través del dinero y el financiamiento de las campańas, e influencia a través de candidatos indoamericanos.

Gupta (2004) por su parte, aborda la relación de India con la diáspora india asentada en los Estados Unidos en cuanto recurso político. Seńala que esa comunidad devino más activa políticamente por su volumen, su concentración en determinadas áreas urbanas metropolitanas, su prosperidad, su conectividad, la madurez política de una nueva generación de indoamericana, el hecho de que algunos familiares de la elite india formen hoy parte de esa migración y distintos eventos críticos que tornaron proactiva a la comunidad en ese país. Rodríguez de la Vega (2015) atiende a su influencia en la política exterior de India y concluye que aunque el alcance de su accionar resulta a su criterio -aún limitado- debido al pequeño volumen de sus votantes, estos emigrados precisan profundizar su accionar en el congreso del país de residencia mediante la apelación a una "diplomacia diaspórica".

Van Hear, Pieke y Vertovec (2004) exploran el papel desempeñado por distintas diásporas asentadas en el Reino Unido en lo que hace al desarrollo y la reducción de la pobreza en sus países de origen -incluido la India-. Menski (2002) estudia cambios en el escenario sociopolítico británico y en las percepciones sobre migración y multiculturalismo. Mohapatra (2012) aborda la participación política de esta diáspora en el Reino Unido y Choudhari (2014), por su parte, se enfoca en la relación entre la diáspora india y la transformación política de la Gran Bretańa actual. Para este autor, la participación electoral de grupos étnicos afectó tanto la naturaleza como el alcance de la política británica a lo largo de los años. En atención a la creciente participación y representación, esa Gran Bretaña multiétnica está en la fase de tránsito desde la "colonización étnica" (Ballard, 1994) a la "colonización política".

Bhargava, Sharma y Salehi (2008) dan cuenta de mesas redondas desarrolladas en Toronto, Nueva Delhi y Chandigarth, acerca de cuestiones relativas a la relación bilateral entre India y Canadá, la visión de los emigrados 
indios residentes en ese país y el potencial de ese segmento de la diáspora para contribuir a estimular la asociación entre ambos países.

Por su parte, Khory (2006) estudia la influencia de las redes tecnológicas en la movilización política transnacional. Se enfoca en la comparación entre los sikh de Punjab y los tamiles de Sri Lanka en relación con la comunicación de sus proyectos de autodeterminación a los emigrados.

Centrados en el subgrupo sikh de la diáspora, Dhillon (2007) atiende el papel desempeñado por la diáspora en el movimiento separatista sikh para la constitución de Khalistán (estado sikh separado). Señala que uno de los aspectos más importantes de esa función se relaciona con la rápida internacionalización de un conflicto interno de la India. Purewal (2012) sostiene que la militancia sikh desapareció de la India a mediados de los noventa, pero aún existe en la diáspora. Indica, asimismo, que a diferencia de lo sucedido anteriormente, en la actualidad Occidente e India acercan posiciones relativas a la cuestión del terrorismo. Singh Kahlon (2012) en su abordaje de los sikhs en América Latina, apunta los datos y su búsqueda personal de una supuesta Embajada de Khalistán asentada en Quito, Ecuador, de la que no encontró rastro alguno.

Jaffrelot y Therwath (2007) acometen el análisis del nacionalismo hindú de larga distancia que cuestiona la naturaleza cambiante del nacionalismo en el mundo global y comparan sus dinámicas en Canadá, Estados Unidos y Reino Unido. Therwath (2012), señala que el centro de las fuerzas nacionalistas hindúes en la diáspora se encuentra en los Estados Unidos, donde además distintas diásporas nacionalistas confluyen en la islamofobia.

Otro tema de interés es el considerado por Ibarra (2012) al tratar las castas como identidades que atraviesan fronteras. Se refiere de modo particular a la Red Internacional de Solidaridad Dalit (IDSN) que busca desarrollar trabajos a escala global para eliminar la discriminación basada en la casta y otras formas similares. En línea con ello, Singh (2015) en su trabajo sobre la casta en la diáspora, hace referencia a espacios institucionales como el encuentro Patidar Samaj llevado a cabo en Atlanta, con el propósito de establecer contacto entre jóvenes de la misma casta con miras al arreglo de casamientos (ello se suma a una estructura organizacional compleja de las diversas asociaciones de los Patidar en el ámbito de la diáspora).

\section{Notas finales}

La relación triádica país de origen-diáspora-país de residencia, encuentra un nutrido cuerpo de estudios que busca abordarla integralmente (los menos) o a uno o más de sus componentes desde la perspectiva de distintas ciencias sociales, con trabajos que enfatizan los abordajes cuantitativos sobre todo al tratar cuestiones económicas.

Este trabajo considera los aportes de distintas disciplinas de las ciencias sociales en la idea de que un abordaje interdisciplinario de la cuestión contribuirá a una mayor comprensión del fenómeno. En ese marco, distintas nociones se destacan en los abordajes descriptos, a saber: Oonk (2007) activa la discusión acerca de los diversos sentidos del término "diáspora" y enfatiza no solamente su no univocidad, sino también la heterogeneidad del fenómeno. Este autor busca aprehender ambas cuestiones, que sin duda son relevantes para cualquier consideración sobre la materia.

En ese marco y de acuerdo con el seńalamiento de Vertovec (Vertovec y Cohen, 1999) de que en la actualidad el término diáspora es usado para referirse a cualquier población desterritorializada o transnacional, Leclerc (2004) y Rodríguez de la Vega (2014) consideran la "invención" de la diáspora india y remiten a lo señalado por Richard (2002) acerca de que el uso de determinada terminología para referirse a un grupo social para reconocerlo como tal, contribuye a investirlo de sentidos específicos, lo que implica el nombre y el modelaje de una categoría de inteligibilidad.

Bhat y Narayan (2010) entienden el transnacionalismo como la extensión por las diásporas de su relación con el país de origen y su conexión con otras comunidades dispersas en el mundo, sumado a los sentimientos comunitarios. Ello, además de la polisemia del término, conecta la dinámica transnacional con la teoría del nacionalismo en general y repasa los conceptos de nación -entendida como una comunidad imaginada-y de "nacionalismo de larga distancia", ambos enunciados por Anderson (1993), así como la idea de "nacionalismo diaspórico" enunciado por Gellner (1988).

En línea con ello, se hace presente la consideración del poder blando, que describe la capacidad que un actor político posee para incidir en las acciones o intereses de otros actores, utilizando medios culturales e ideológicos con complementos de medios diplomáticos, y enfatiza las posibilidades de cooperación (Nye, 2004). De igual modo, se atiende a la diplomacia pública, que en un sentido general alude a la comunicación con públicos foráneos para establecer un diálogo que permita informar al tiempo que influenciar. Esta comunicación se puede dar en dos modalidades: la diplomacia pública "gente a 
gente" y el "gobierno a gente" (Mannheim, 1994 citado en Noya, 2006).

Lacroix (2011), por su parte, deja planteada la cuestión de los "campos organizacionales transnacionales", que al igual que las búsquedas de Rodríguez de la Vega (2014) sobre la reconfiguración del tiempo y espacio entre el país de origen y la diáspora y los paralelos de Naujoks (2010) entre práctica política y producción de conocimiento sobre la diáspora, merecen mayor exploración. Del mismo modo, en relación con los segmentos de la diáspora considerados en los estudios sobre esta temática, cabe señalar que existe mayor producción sobre algunos de los segmentos más poblados de la diáspora (Estados Unidos, Reino Unido, etc.) y una notoria escasa presencia de trabajos sobre segmentos diaspóricos de menor volumen poblacional, como los asentados en Latinoamérica.

Para finalizar, se comparte y propone extender la propuesta de Omer (2011) en lo referido a una retroalimentación entre estudios de las diásporas en las relaciones internacionales y estudios sobre ellas desde otras perspectivas de las ciencias sociales, en consideración a la presencia de otros agentes en la arena internacional que contribuyen a complejizar las relaciones en ese ámbito y a la importancia de la cultura en las relaciones internacionales, todo con miras a enriquecer el aparato conceptual que orienta los análisis y las prácticas en lo que hace a las diásporas en sí, al ámbito internacional y a las diásporas en el ámbito internacional.

\section{Referencias}

Abraham, R. (2012). India and its Diaspora in the Arab Gulf Countries: Tapping into Effective 'Soft Power' and Related Public Diplomacy. Diaspora Studies, 5(2), 124-146.

Agarwala, R. (2012). Tapping the Indian Diaspora for Indian Development. In A. Portes \& P. Fernández - Kelly, (Eds.). The State and the grassroots: Inmigrant Transnational Organization in four continentes (pp. 85-110). NY: Berghahn Books.

Anderson, B. (1993). Comunidades Imaginadas. Reflexiones sobre el origen y la difusión del nacionalismo. México: Fondo de Cultura Económica.

Bal, E., \& Sinha-Kerkhof, K. (2007). No "holy cows" in Surinam: India, comunal relations, identity politics, and the Hindostani Diaspora in Surinam. SACS, 1(2), 17-35.

Balasubramanyam, V. N. (2007). The Diaspora and Development. Recuperado de http://goo.gl/RaUKBc

Ballard, R. (Ed.) (1994). Desh pardesh. The South Asian presence in Britain. London: Hurst \& Co.
Bhargava, K., Sharma, J. C., \& Salehi, S. (2008). Building Bridges: A Case Study on The Role of the Indian Diaspora in Canada. Ontario: The Centre for the Study of Democracy.

Bhat, Ch., \& Narayan, K. L. (2010). Indian Diaspora, Globalization and Transnational Networks: The South African Context. J. Soc. Sci, 25 (1-2-3), 13-23.

Bhatt, Ch., \& Mukta, P. (2000). Hindutva in the West: mapping the antinomies of diaspora nationalism. Ethnic and Racial Studies, 23(3), 407-441.

Chand, M. (2009). How does the Indian diáspora help drive trade and investment ties between India and North America? An exploratory Study (Tesis de doctorado Inédita). Simon Fraser University, Canada.

Chanda, R. (2008). The Skilled South Asian Diaspora and its role in source economies. South Asian Diaspora. Syngapore: ISAS.

Chanda, R., \& Sreenivasan, N. (2006). India's Experience with Skilled Migration. In C. Kuptsch \& E. F. Pang (Eds.), Competing for Global Talent (pp. 215-256). Geneva: International Institute for Labor Studies.

Choudhari, S. K. (2014). Political colonization of multi-ethnic Britain: a study of Indian Diaspora. Diaspora Studies, 7(2), 100-120.

Dhillon, S. (2007). The Sikh Diaspora and the Quest for Khalistan: A Search for Statehood or for Self-preservation? New Delhi: Institute of Peace and Conflict Studies.

Gautam, M. K. (2013). Indian Diaspora: Ethnicity and Diasporic Identity. Italia: European University Institute.

Gayer, L. (2007). The Volatility of the 'Other': Identity Formation and Social Interaction in Diasporic Environments. South Asia Multidisciplinary Academic Journal, 1. doi: 10.4000/samaj.36

Gellner, E. (1988). Naciones y Nacionalismo. Madrid: Alianza.

Gottschlisch, P. (2008). The Indian Diaspora in the United States of America: An Emerging Political Force? In P. Raghuram, A. K. Sahoo, B. Maharaj \& D. Sangha, D. (Eds.), Tracing an Indian diaspora. Contexts, Memories, Representations (pp.156-170). New Delhi: Sage.

Gupta, A. (2004). The Indian Diaspora's Political Efforts in the United States. New Delhi: Observer Research Foundation.

Gupta, R. K. (2013). Non.State actors in international relations: a case of Indian diáspora in Africa. Recuperado de http:// goo.gl/8xzjBt

Heath, D., \& Mathur, Ch. (Eds.) (2011). Communalism and globalization in South Asia and its diáspora. London/NY: Routledge.

Hercog, M., \& Siegel, M. (2010). Engaging the diaspora in India (Working Paper Series No. 2011-041). The Netherlands: Maastrich Graduate School of Governance. 
Ibarra, L. (Septiembre. 2014). Castas: Identidades que atraviesan fronteras. Trabajo presentado en el Seminario "Perspectivas de cultura y política en la India. Buenos Aires, Argentina.

Jaffrelot, Ch., \& Therwath, I. (2007). The Sangh Parivar and the Hindu Diaspora in the West: What Kind of "LongDistance Nationalism"? International Political Sociology, 1(3), 278-295.

Janardhanam, V. (2013). Political participation of the Indian Diaspora in the USA. Journal of International and Global Studies, 5(1), 16-33.

Kapoor (2003), The Indian Diaspora as a Strategic Asset. Economic and Political Weekly, 38(5), 1-7.

Kapur, D. (2010). Diaspora, development and democracy. The domestic impact of international migration from India. NJ: Princeton University Press.

Khanna, P. (2005). Bollystan: India’s Diasporic Diplomacy. In P. K. Basu, B. Chellaney, P. Khanna \& S. Khilnani, S. (Eds.), India as a New Global Leader (pp. 16-26). London: Foreign Policy Centre.

Khory, K. R. (2006). Diaspora Populations, the Internet and olitical Mobilization. In S. Rajagopalan (Ed.), Security and South Asia. Ideas, Institutions and Initiatives (pp. 152-175). New Delhi: Routledge.

Kinnvall, C., \& Svensson, T. (2010). Hindu nationalism, diaspora politics and nation-building in India. Australian Journal of International Affairs, 64(3), 274-292.

Lacroix, T. (2011). Indian and Polish Migrant Organisations in the UK (HA archives ouvertes No. halshs- 00820303). Oxford: IMI.

Leclerc, E. (Octubre, 2004). Linvention d'une diaspora indienne: enjeux politiques et sociaux. Trabajo presentado en Espaces et sociétés aujourd'hui (la géographie sociale dans l'espace et dans l'action). Université de Rennes, Rennes-France.

Leclerc, E. (2008). India recentred: The role of Indian diáspora in the globalisation process (Working Paper No. 2). New Delhi: IMDS.

Mason, R. (2013). Realizing the Indo-Saudi "Strategic Partnership": An Analysis of the Leading Drivers. In R. Gupta, A. B. Bagader, T. Ahmad \& N. Janardhan (Eds.), India and the Gulf: What Next? (pp. 103.121). Cambridge: Gulf Research Centre Cambridge.

Menski, W. F. (Octubre, 2002). Immigration and multiculturalism in Britain: New issues in research and policy. Trabajo presentado en Osaka University of Foreign Studies, Japon.

MEA. (2002). Report of the High Level Committee on Indian Diaspora. New Delhi: Indian Council of World Affairs.

Mitchell, M. I. (Junio, 2012). The Perils of Population Movements in International relations: new directions for rethinking the migration-conflict nexus. Trabajo presentado en el Canadian Political Science Association Annual Meeting. Edmonton, Canada.

Mohammad-Arif, A. (2007). The Paradox of Religion: The (re)Construction of Hindu and Muslim Identities amongst South Asian Diasporas in the United States. South Asia Multidisciplinary Academic Journal, 1. doi: 10.4000/samaj.55

Mohammad-Arif, A. (2000). The Lobbying Game of the Indian and Pakistani Diasporas in the U.S (Working Paper for Le Dossier). Paris: Centre International Studies and research.

Mohan, C. R., \& Chauhan, R. (2015). Modis Foreign Policy: Focus on the Diaspora (ISAS Working Paper). Singapore: Institute of South Asian Studies.

Mohapatra. (Febrero, 2012). Indian Diaspora and Political Participation in United Kingdom-Issues o Perspectives. Trabajo presentado en la International Conference on Empire, Nation and Diaspora: Mapping the Trajectories of Transformations in Indian Diaspora. Hyderabad Central University, India.

Mulloo, A. (2007). Voices of the Indian Diaspora. Delhi: Motilal Banarsidass.

Murti, B. (2013). India's Use of Digital Media to Engage with Diaspora as Part of its Public Diplomacy Outreach a case study analysis of the website of Overseas Indian Facilitation Centre (Tesis de Maestría inédita). The Faculty of the Public Communication Program School of Communication American University Washington, D.C.

Naujoks, D. (2010). India and its Diaspora. Changing Research and Policy Paradigms. In D. Thränhardt \& M. Bommes (Eds.), National Paradigms of Migration Research (pp. 269300). Göttingen: V\&R Unipress.

Niumai, A. (2009). Philanthropy and Indian Diaspora in the 21st century. Trabajo presentado en el Centre for Research on Nationalism, Ethnicity and Multiculturalism. University of Surrey, UK.

Noya, J. (2006). Una diplomacia pública para España (Documento de trabajo No. 11-2006). Madrid: Real Instituto Elcano.

Nye, J. (2004). Soft Power. NY: PublicAffairs.

Omer, A. (2011). Rethinking "home" abroad: religión and the reinterpretation of national boundaries in the Indian and Jewish Diasporas in the U.S. International Journal of Peace Studies, 16(1), 23-52.

Oonk, G. (Ed.) (2007). Global Indian Diasporas: Exploring Trajectories of Migration and Theory. Amsterdam: Amsterdam University Press.

OIM. (2010). Previsiones y Tendencias Mundiales. Recuperado de http://www.newwebsite.iom.int

$96<$ Universidad de San Buenaventura, Cali - Colombia 
Pandey, A., Aggarwal, A., Devane, R., \& Kuznetsov, Y. (2004). India's Transformation to Knowledge-based Economy Evolving Role of the Indian Diaspora. Evalueserve. Recuperado de http://goo.gl/2WhgMx

Prashad, V. (2003). Namaste Sharon: Hindutva and Sharonism under U.S. Hegemony. New Delhi: LeftWord Books.

Purewal, S. (2012). The Evolution of Sikh Secessionist Movement in western Liberal Demoracies. International Journal of Business and Social Sciences, 3(18), 107-113.

Reddy, M. (2015). Social Movements and the Indian Diaspora. Routledge.

Richard, N. (2002). Saberes Académicos y Reflexión Crítica en América Latina. En D. Mato (Comp.), Estudios y otras prácticas intelectuales latinoamericanas en cultura y poder (pp.363-372). Caracas: CLACSO.

Rodriguez de la Vega, L. (2014). La cartografía de la movilidad: la diáspora en el marco de la articulación entre lo local y lo global. Journal de Ciencias Sociales, 1(2), 3-15.

Rodriguez de la Vega, L. (2015). Las diásporas en la arena internacional: el caso de la diáspora india. Oasis, 22, 23-47. doi: http://dx.doi.org/10.18601/16577558.n22.03

Roy, J., \& Banerjee, P (2007). Attracting FDI from the Indian Diaspora: The Way Forward. Recuperado de http://goo.gl/ jmgSxg

Sharma, J. (s.f.) Características de la diáspora india y su relación con el país de origen. Recuperado de http://goo.gl/ZZZloF

Sheffer, G. (Ed.) (1986). Modern Diasporas in International Politics. Australia: Croom Helm Ltd.
Singh, A. D. (2012). Working with the Diaspora for Development. Policy Perspectives from India. New Delhi: Indian Institute of Management Bangalore.

Singh, S. (2005). Sending Money Home: Money and Family in the Indian Diaspora, Recuperado de http://goo.gl/GBHau4

Singh, S. (2012). Sikhs in Latin America. Travels among the Sikh Diaspora. New Delhi: Manohar.

Sing, S. (2015). Caste and Diaspora. International Journal of Social Science and Humanity, 5(1), 80-82.

Therwath, I. (2007). Working for India or against Islam? Islamophobia in Indian American Lobbies. South Asia Multidisciplinary Academic Journal, 1. doi: 10.4000/samaj.262

Therwath, I. (2010). 'Shining Indians': Diaspora and Exemplarity in Bollywood. South Asia Multidisciplinary Academic Journal, 1. doi: 10.4000/samaj.3000

Therwath, I. (2012). Cyber-Hindutva: Hindu Nationalism, the diaspora and the web. New Delhi: Centre de Sciences Humaines.

Varadarajan, L. (2010). The Domestic Abroad. Diasporas in International Relations. NY: Oxford University Press.

Van Hear, N., Pieke, F., \& Vertovec, S. (2004). The contribution of $U K$-based diásporas to development and poverty reduction. Recuperado de http://goo.gl/GBHau4

Vertovec, S., \& Cohen, R. (1999). Migration, Diasporas and Transnationalism. Massachusetts: Edward Elgar Publishing Limited.

Ziqun, Z. (2007). Two Diasporas: Overseas Chinese and Nonresident Indians and Their Homelands' Political Economy. Journal of Chinese Political Science, 12, 281-296. 